\title{
Characteristics Analysis of External Treatment of Chinese Medicine for Constipation
}

\author{
Rui Zhang ${ }^{1, a, *}$ and Mingsan Miao ${ }^{1, b}$ \\ ${ }^{1}$ Henan University of Chinese Medicine, Pharmacology subject, Zhengzhou, Henan, China \\ azhangruiyaoli@126.com, ${ }^{b}$ miaomingsan@163.com \\ *corresponding author
}

Keywords: Constipation, Chinese Medicine External Treatment, Characteristics

\begin{abstract}
Constipation is a common digestive tract disease. It is mainly manifested as difficulty in defecation, less defecation, accompanied by or without defecation. The etiology is complex and diverse, and the treatment factors are also very extensive, which seriously affects people's lives and health. At present, the external application of traditional Chinese medicine is widely used to treat constipation, avoiding the toxic side effects of Western medicine treatment. Besides, Chinese herbal medicine does not bring tolerance and laziness to patients. This article analyzes the characteristics of constipation treated with Chinese medicine external treatment.
\end{abstract}

\section{Introduction}

At present, the prevalence of constipation is increasing year by year. The characteristic is that with the increase of age, women are more than men, and there are more in cold, poor and rural areas. Constipation brings a lot of dangers to the human body. It mainly includes four aspects: aggravating cardiovascular and cerebrovascular diseases, leading to self poisoning, causing perianal infection, causing negative emotions and psychological and social dysfunction. In recent years, western medicine always uses laxatives to constipation, easy to produce tolerance and dependence. Chinese medicine treatment of constipation has incomparable advantages of Western medicine. Chinese medicine external treatment of this disease has become the focus of the study of physicians. Based on the clinical and experimental study of Chinese medicine external treatment in the past ten years in China's CNKI (2007-2017), this paper analyzes the characteristics of Chinese medicine external therapy in clinical application, and provide some guidance for the research and development of diagnosis and treatment of constipation clinical drugs.

\section{Chinese Medicine Etiology and Treatment of Constipation}

Constipation can be caused by many factors, and it is a symptom of many diseases. Chinese medicine believes that constipation is caused by intestinal conduction dereliction of duty. It is also related to the dysfunction of the viscera [1]. Fecal retention in the intestines is too long, constipation is illogical, defecation cycle extended, or cycle is not long, but the fecal matter dry scleroma. Chinese Medicine thinks that constipation is related to Qi deficiency, liver depression, kidney deficiency or improper diet. Treatment of traditional Chinese medicine advocates treatment from the root. It usually uses the methods of Purging heat, tonifying qi, enriching the blood and moisturizing the intestine and regulating qi to treat constipation. 
Table 1 Dialectical treatment of constipation in traditional Chinese medicine

\begin{tabular}{|c|c|c|}
\hline Disease type & Symptom & Therapeutic principle \\
\hline Bowel heat & $\begin{array}{l}\text { Abdominal pain, Dry mouth halitosis, Urine short } \\
\text { red, Red tongue, Pulse slippery number. }\end{array}$ & $\begin{array}{l}\text { The main treatment method is } \\
\text { purgingheat. }\end{array}$ \\
\hline $\begin{array}{l}\text { Liver temper } \\
\text { stagnation }\end{array}$ & $\begin{array}{l}\text { Dry feces, Pain in the abdomen, Fullness of the chest } \\
\text { and abdomen, Thin and greasy tongue fur, wiry } \\
\text { pulse. }\end{array}$ & $\begin{array}{l}\text { The main treatment method is } \\
\text { guiding qi downward. }\end{array}$ \\
\hline $\begin{array}{l}\text { Yin } \\
\text { deficiency } \\
\text { inner heat }\end{array}$ & $\begin{array}{l}\text { Repeated stool constipate, Shortness of breath, } \\
\text { Abdominal cold, mental fatigue, pale tongue, thin } \\
\text { weak pulse. }\end{array}$ & $\begin{array}{l}\text { The main treatment method is } \\
\text { warming interior to dissipate cold. }\end{array}$ \\
\hline $\begin{array}{l}\text { deficient qi } \\
\text { and blood }\end{array}$ & $\begin{array}{l}\text { Difficult defecation, Lusterless complexion, Pale } \\
\text { tongue, Weak pulse. }\end{array}$ & $\begin{array}{l}\text { The main treatment method is } \\
\text { invigorating qi and blood }\end{array}$ \\
\hline $\begin{array}{l}\text { Kidney yang } \\
\text { deficiency }\end{array}$ & $\begin{array}{l}\text { Dry stool, Urine clear long, Pale complexion, Limbs } \\
\text { not warm, Cold pain in the abdomen, Lumbar acid } \\
\text { cold, Pale tongue with white fur, Pulse Shen chi. }\end{array}$ & $\begin{array}{l}\text { The main treatment method is } \\
\text { warming yang to promot defecation. }\end{array}$ \\
\hline $\begin{array}{l}\text { Congestion } \\
\text { retardation }\end{array}$ & Qi stagnation and blood stasis & $\begin{array}{l}\text { The main treatment method is } \\
\text { promoting blood circulation for } \\
\text { removing blood stasis. }\end{array}$ \\
\hline
\end{tabular}

\section{Traditional Chinese Medicine Treatment Characteristics of Constipation}

\section{A. Point Application}

Traditional Chinese medicine acupoint sticking therapy has a long history, exact curative effect, high economic benefit and little adverse reaction. It has unique advantages and significant curative effect. The main acupoints of acupoint application is Shenque [2]. The secondary acupoints is Zhongwan, Dachangshu, Tianshu, Qihai, Guan Yuan, etc. The excipients of acupoint application is mainly Vinegar, mashed garlic, ginger juice, honey, edible oil, glycerin, ethanol, etc.

In clinic, we commonly used acupoint application combined with oral Chinese Medicine to treat Constipation. Although acupoint application is rapid and effective, but the preparation is complex and the medication is relatively fixed. Oral medicine can make up for these deficiencies, both complement each other and play a synergistic role [3]. Acupoint application combined with massage can strengthen the peristalsis of the gastrointestinal tract. Acupoint application combined with cupping jar can make the blood vessels dilate in the umbilicus, promote the absorption of drugs, regulate the function of splanchnic nerve, and enhance the digestive function. There are abundant branches of meridians and abdominal arteries under the umbilicus, and there is no accumulation of cholesterol. The barrier is weak and permeability is strong, which is beneficial to drug absorption. The drug was absorbed directly by acupoint. The doctors select Shenque, bilateral Tianshu, bilateral Dachangshu, using the amount of yanhua, euphorbia, gansui, jujube to make powder. Then it was made of ointment paste with mel. Finally, they use it to paste on Shenque point. 100 cases of this disease were treat. The total effective rate was $92.9 \%$.

\section{B. Catgut Implantation at Acupuncture Point}

In order to produce lasting acupoint stimulation, Catgut embedding therapy often uses catgut to stimulate acupoints. It through recuperating viscera, balancing yin and yang, So as to play a therapeutic role. Catgut embedding therapy use stimulation mode of soft and lasting to harmonize qi and blood and dredge meridian for treating constipation.

LinShu·ZhongShi said, " Evil deep inside, Stab this patient, expelling Pathogenic factors" [4]. The 
acupoint catgut embedding therapy integrates acupuncture effect, catgut embedding effect and pricking blood therap and its clinical therapeutic effect is remarkable [5]. The use of catgut can produce longlasting stimulation on the related points (usually 20 days). It can promote intestinal smooth muscle excitability and increase peristalsis. In the clinic, it is often used in the treatment of idiopathic constipation and habitual constipation [6]. The main acupoints of treatment of Primary constipation is Stomach meridian, bladder meridian, spleen meridian. The main acupoints of treatment of habitual constipation is Tianshu, Zhaohai, Lieque, Dachangshu, Zhigou, Zusanli. The clinical selects Tianshu, Daheng, Shangjuxu. It uses catgut to stab subcutaneous tissue of acupoints at Tianshu, crossing through the Daheng. Finally, it is embedding the catgut in Shangjuxu. The effective rate was 94.30\% [7].

Catgut embedding therapy for patients with long-term constipation can reduce times of treatment of patients. The patient is easy to accept, and its less pain. Clinical practice shows that catgut implantation at acupoint can improve sphincter, pelvic floor muscle and lower anal canal pressure [8]. In recent years, acupoint catgut embedding for treatment of constipation includes simple acupoint catgut embedding therapy, acupoint catgut embedding therapy combined with Chinese medicine treatment, acupuncture combined with acupuncture and massage therapy, acupoint catgut embedding therapy combined with auricular therapy, acupoint catgut embedding therapy combined with traditional Chinese medicine method of umbilicus [9].

\section{Moxibustion Therapy}

Moxibustion treatment of constipation is reliable, easy to operate, no side effects and adverse reactions, and has been a common method of clinical treatment of constipation. The essential component of Folium Artemisiae argyi is essential oil [10]. The volatile oil produced by moxa leaf combustion has antibacterial and bactericidal effects. It can inhibit staphylococcus aureus, beta streptococcus, escherichia coli and pseudomonas aeruginosa. Moxibustion treatment of constipation, its nature is mild, which can penetrate the skin, directly to the deep tissue. Moxibustion through the surface acupoint into the body and the meridians [11]. It can dilate the lumen of capillaries, promote blood and lymph circulation, and increase intestinal fluid secretion, contribute to the evacuation of the bowels. This method is accepted by the patients and easy to learn, use and popularize.

\section{Auricular Therapy}

Auricular therapy for the treatment of constipation include ear acupuncture and auricular plaster. Acupoint sticking is based on the theory of Zang. LinShu says "The ear is the gathering place of meridians". Auricular points have direct and indirect connections with the twelve meridians, and the nerve distribution of the auricle is very rich [12]. Each organ in the human body corresponds to the specific area of the auricle [13]. According to the research, it can stimulate the human parasympathetic nerve to promote gastrointestinal peristalsis by stimulating the nerves under the auricular points [14]. The ear acupoints, often in the large intestine, rectum and Sanjiao acupoints. Auricular Vaccaria seed is commonly used materials, followed by magnetic beads, radish seed, rapeseed, tea seed, mung bean and liushenwan.

Auricular point therapy for constipation, has a wide range clinical application, good curative effect convenient operation, good compliance and no adverse reactions [15][16]. It can avoid the damage of kidney function caused by taking various laxatives, or the decrease of intestinal peristalsis, the decrease of spontaneous defecation reflex and the relaxation of anal sphincter caused by clysma.

\section{Summary}

Nowadays, the rhythm of life has brought changes to the diet structure, and the incidence of constipation in the population is increasing year by year. Constipation can bring obstacles to life and mental health. If not timely attention, prevention and control. This can cause the accumulation of endotoxin, diarrhea, 
abdominal pain, and serious gastrointestinal nerve dysfunction. And it will have a certain impact on the cardiovascular and cerebrovascular system, urinary and reproductive system. In recent years, according to the clinical practice and observation, treatment of constipation with Chinese medicine external therapy has good curative effect. Although Chinese medicine treatment of constipation in different ways, but its curative effect, and no toxic side effects, easy to be accepted by patients, compared with western medicine or oral Chinese medicine has obvious advantages. External treatment of Chinese medicine can avoid these side effects, the treatment is simple and convenient, the treatment effect is remarkable, easy to promote. Constipation of traditional Chinese medicine external treatment, mainly through the meridians and acupoints, adjusting the internal organs, dredging meridians, regulate qi and blood, balance of yin and yang to promote gastrointestinal peristalsis, improve its excitability, increase intestinal fluid secretion, relieve the intestinal spasm. External treatment can avoid the absorption of digestive enzymes and liver in vivo, and avoid the influence on gastrointestinal tract. At present, for the treatment of constipation, acupoint application, acupoint catgut embedding and acupuncture have achieved very good curative effect, and are mostly used in clinic.

The development of TCM external treatment constipation is very large, but there are many problems in its development process. Although the clinical effect is very good, but also is clinical observation and experience. Faced with this situation, we should strengthen the basic, systematic and in-depth experimental research. Broaden the clinical and basic experimental research on the treatment of functional constipation with Chinese Medicine External therapy. Focus on further study from the theoretical mechanism, external therapy treatment plan design, operation methods, drug compatibility, excipient selection, reasonable treatment time. In the clinical research, we should increase the pathological inclusion criteria and the basis of curative effect judgment, so that the clinical treatment results are sufficiently convincing and reliable.

\section{Acknowledgment}

Fund Project: National "11th Five-Year" support program (2008BAI53B09), national traditional Chinese medicine administration of traditional Chinese medicine Standardization (2017-149-11).

\section{References}

[1] Zhang Rongzhi, Qian haihua. Analysis of etiology and treatment of constipation [J]. Medical summary, 2014, 20 (01): 118-120. [2017-09-04].

[2] Zhang Yueqi, Dong Fangfang, Wang Kejie. Etiological analysis and research progress of constipation [J]. Henan Journal of preventive $\mathrm{m}$

[3] edicine, 2012, 23 (03): 248-250. [2017-09-04]. DOI: 10.13515/j.cnki.hnjpm.2012.03.044

[4] Qu Meng, Ma Xiaoli. Clinical research progress of acupoint sticking therapy for constipation [J]. Medical research and education, 2013, 30 (02): 76-81. [2017-09-04].

[5] Ma Liya, Zhu Yin, Lu Ying, Li Jie. Research progress of catgut embedding therapy for constipation [J]. Jilin Journal of Traditional Chinese Medicine, 2016, 36 (04): 426-429. [2017-09-04]. DOI: 10.13463/j.cnki.jlzyy.2016.04.032

[6] Jin Fujie, Meng Hongyan. Clinical research progress of external treatment of Chinese medicine for constipation in children [J]. Journal of Clinical Acupuncture and Moxibustion, 2013, 29 (04): 70-73. [2017-09-03].

[7] Chen Pingping, Chen Yuyan. Research progress of external treatment of Chinese medicine for functional constipation in children $[\mathrm{J}]$. Journal of Shaanxi University of traditional Chinese Medicine, 2016, 39 (03): 114-116. [2017-09-03].

[8] Liu Weifeng, Jiang Yuping, Huang Yongguang, Huang Mingtu, Huang Zaiping. Research progress 
of external treatment of Chinese medicine for constipation [J]. Journal of external treatment of traditional Chinese medicine, 2014, 23 (01): 51-52. [2017-09-03].

[9] Zhou Yuchen, Bai Guang. General situation of functional constipation treated by external therapy [J]. Journal of Practical Traditional Chinese Internal Medicine, 2013,27 (22): 73-75. [2017-09-03].

[10] Zhou Lifeng, Shi Shaoyan, Yao Meimei. Application progress of external treatment of Chinese medicine for constipation caused by opioids [J]. Journal of clinical rational drug use, 2015, 8 (15): 177179. [2017-09-03].

[11] Cai Jinghua, Zhang Xingzhen, Zhang Yongchen. Research progress of external treatment of traditional Chinese medicine for habitual constipation [J]. Hunan Journal of traditional Chinese medicine, 2014, 30 (06): 163-164. [2017-09-03].

[12] Yang Lihua, Duan Beipei. Application of external therapy in opioid induced constipation [J]. Journal of Liaoning University of Traditional Chinese Medicine, 2013, 15 (04): 246-248. [2017-09-03].

[13] Chen Yajuan, Zhu Yanan, Luo Yan, Zheng Jianfen, Hu Zhuhong. Research Progress on external treatment of diabetic constipation with Chinese Medicine [J]. new Chinese medicine, 2014, 46 (04): 211 -214. [2017-09-03].

[14] Luo Fu, Wang Yipei, Zhu Ying. Research Progress on external treatment of functional constipation with Chinese Medicine [J]. Guiding Journal of Traditional Chinese Medicine and Pharmacology, 2017, 23 (02): 86-88. [2017-09-03]. DOI: 10.13862/j.cnki.cn43-1446/r.2017.02.025

[15] Li Ying, Zhong Ning, Hua Yu. Treatment of functional constipation with TCM External Therapy [J]. Shanghai medicine, 2013, 34 (18): 37-40. [2017-09-03].

[16] Feng Shuo, Liu Shaoneng. Research Progress on Mechanism of functional constipation treated by traditional Chinese Medicine [J]. Chinese digest of the world, 2013, 21 (06): 459-463. [2017-09-04].

[17] Jia Fei, Qiu Jianfeng, Yuan Liang, Li Guofeng, Shu Tao, Hong Zifu, Li Guodong. Recent research on external treatment of constipation with TCM External Therapy [J]. Jilin Journal of Traditional Chinese Medicine, 2007, (03): 60-62. 